\title{
Understanding the Everyday Use of Head-Worn Computers
}

\author{
Anita Vogl ${ }^{1}$, Nicolas Louveton ${ }^{2}$, Rod McCall ${ }^{2}$, Mark Billinghurst ${ }^{3}$, Michael Haller ${ }^{1}$ \\ ${ }^{1}$ Media Interaction Lab \\ University of Applied Sciences Upper \\ Austria \\ Hagenberg, Austria \\ mi-lab@fh-hagenberg.at \\ ${ }^{2}$ Interdisciplinary Centre for Security, \\ Reliability and Trust Weicker Building \\ Université du Luxembourg \\ Luxembourg \\ $<$ firstname $>$.<lastname>@uni.lu \\ ${ }^{3}$ HIT Lab NZ \\ University of Canterbury \\ Canterbury, New Zealand \\ mark.billinghurst@hitlabnz.org
}

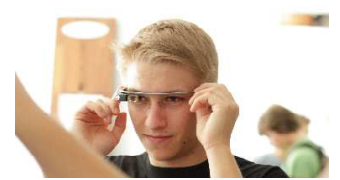

(a) Setting up a HWC.

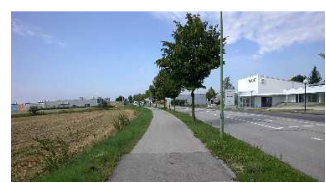

(b) Taking a picture on the bike.

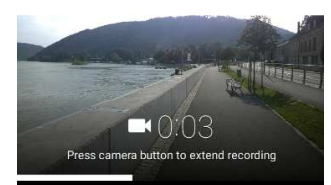

(c) Recording a video on-the go.

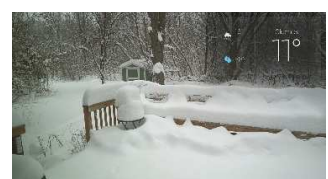

(d) Checking the weather in winter.

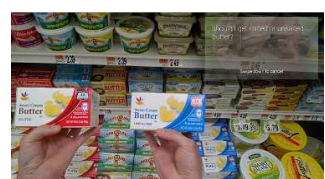

(e) Messaging during shopping.

Fig. 1.: Use cases for head-worn computers.

\begin{abstract}
Early research on head-worn computers (HWCs) has focused on hardware and specific applications. However, there is little research about the everyday usage of head-worn computers in particular aspects such as: context of use, social acceptance across different activities, audiences and interaction techniques. This paper provides insights into the use of headworn computers by capturing the opinions of novice and expert users through a survey, a three-week diary study, and interviews. The overarching finding is that the context of use is critical, either due to the need to support micro-interactions, or because the interaction paradigm itself should depend on the context of use.
\end{abstract}

Keywords-head-worn computers; smart glasses; everyday use; social acceptability; interaction techniques

\section{INTRODUCTION}

Since the introduction of the first head-mounted displays [1] the landscape of head-worn computers has evolved. With the introduction of Google Glass ${ }^{1}$, Epson Moverio ${ }^{2}$, and other small form-factor devices there is an emergence of HeadWorn Computers (HWC) in recent times. These could be more widely used in the consumer market in the next few years due to their small form factor and wearability. These consumer HWCs have onboard microphones, small capacitive input sensors, and voice recognition for smooth command-based interaction, bringing a new experience of digital information directly to the user. According to Gartner Research, wearable user interfaces in general will reach its plateau of productivity in the next five to ten years ${ }^{3}$; hence, it is likely that these devices currently mainly available for early adopters will reach the whole market in the next few years.

Of particular interest to the authors is the use of HWCs within everyday contexts, where people are likely to use such technologies for everyday tasks, in everyday environments, on

\footnotetext{
${ }^{1}$ https://www.google.com/glass/start/

${ }^{2}$ http://www.epson.de/moverio

${ }^{3}$ http://www.gartner.com/newsroom/id/2819918
}

their own, or within social settings. This is often in stark contrast to where HWCs are used. Often such studies take place specifically using applications that are designed for relatively short usage periods (cf. TimeWARP [2]) or in very specific contexts of use where the work practice itself is likely to be quite tolerant, for example, architectural or engineering applications. While social considerations need to be taken into account within such environments the restricted use and/or novelty factor of such experiences can negate some longerterm concerns about acceptance. Indeed longer-term acceptance is often not something that can be grasped within such relatively short studies.

The goal of this paper is therefore to provide a detailed picture of how users are using HWCs nowadays. More explicitly, we studied when, where, why, and how people interact with HWCs. We investigated what these devices are used for and how they support users in their daily life. Additionally, we explored the location of use and studied contextual factors, which influence device usage. Our research employed the combination of a survey, interviews, and a diary study. The investigation took place during May - August 2014 with participants from North America, Europe, and Asia.

In summary, our contributions are as follows:

- We present an in-depth investigation of the everyday use of HWCs. In contrast to previous work, we focus on how these devices support the daily life of a usual consumer rather than specialists.

- We provide a detailed picture of the activities performed with HWCs. We provide insights on what all these activities have in common, how the device changes users' behavior and why we should design for micro-interactions.

- We explore the location of use of HWCs. We show the acceptance of the device in different spaces and explore the acceptability of using the device around different audiences. We illustrate that HWCs are appropriate for environments, where other devices are disadvantageous and why we should design for context. 
- And finally, we investigate the current interaction techniques of HWCs. We discuss current problems and limitations and explain why hands-free interaction is one of the key benefits of the devices.

\section{RELATED WORK}

HWCs are different from mainstream wearable computers in a number of ways, including having a different form factor, interaction metaphors and input techniques, and combining all display, sensing, processing and input technologies into one head-worn unit. These differences mean that HWCs should be studied separately from other examples of wearable systems. Google Glass is the most well-known example of a head-worn computer, but there are other commercially available examples such as Recon $\mathrm{Snow}^{4}$, Vuzix M-100 ${ }^{5}$, or Opinvent Ora ${ }^{6}$.

While head-worn displays and wearable computers are well established research topics and by many researchers in different areas [3]-[5], many of those studies concentrated on perceptual issues [6]-[8] and on work-related tasks with long and potentially complex interactions [9]-[11]. Until recently, only a few studies addressed user related issues: According to Dünser et al. [12], in a survey ranging from 1992-2007, only $10 \%$ of augmented reality researchers involved users in the study; additionally, Swan et al. [13] proposed a similar survey and found that out of 1104 articles 3\% addressed HCI issues and $2 \%$ involved user-tasks. Consequently, there has been little in-depth investigation on the everyday use of HWCs.

\section{A. Everyday Use}

The increasing relevance of context within the design of IT systems can be found within the rising popularity of approaches such as contextual design [14] or the wider field of ethnographic studies of IT. Such approaches look not only at using such methods to capture requirements of such systems but also seek to examine the impact of such systems on the existing context. Extending this to head-worn computers, there some early reports ${ }^{7,8}$ of people being attacked for wearing Google Glass. While the reasons vary, these incidents point to the need to consider where and when HWCs should be worn.

Prior to the current attention given to HWCs, in 1999, Starner [15] presented a landscape of possibilities of wearable computers for the normal, civilian daily life of humans. He describes different scenarios, and points out the advantages as well as the risks of using such devices. All of the presented activities can be performed quickly and support users in the moment they make use of it. According to his work, using such a device combines a lot of functionalities. Lyons [16] presented a case study of an expert user in 2003. He showed that the computer usage plays a secondary role and the device itself enhances the real world experience rather than disturbing it. Since then, devices and in particular the usage context has

\footnotetext{
${ }^{4}$ http://reconinstruments.com/products/jet/

${ }^{5}$ http://www.vuzix.com/consumer/products_m100/

${ }^{6}$ http://optinvent.com

${ }^{7}$ http://mashable.com/2014/02/26/google-glass-assault/

${ }^{8}$ http://mashable.com/2014/04/13/google-glass-wearer-attacked/
}

evolved. Only Pedersen and Trueman [17] discuss briefly the use of Google Glass in everyday life.

In contrast to the little research concerning the everyday use of HWCs over the past few years, there has been substantial research conducted around tablets, smartphones, and other mobile devices which is relevant to the use of HWCs. Müller et al. [18] reported that $41 \%$ of all activities on the tablet were performed while being engaged in another activity. McGregor et al. [19] found that smartphones are mainly used for checking updates or incoming messages (so called micro-breaks), and for creating or consuming content. Böhmer et al. [20] studied smartphone usage in meetings and presented an application for raising people's attention in meetings. In a more psychological study, Oulasvirta et al. [21] found evidence that mobile devices are habit-forming and that the typical checking behavior is reinforced by informational rewards that are easily accessible. Sohn et al. [22] investigated mobile information requests and found that around $72 \%$ of the tasks related to information requests were triggered by their context. Those "micro-breaks", also known as micro-interactions, supporting user activity in a given context are inherent to mobile/wearable computing [23]. While supporting user's routine activity, mobile device usage will interrupt the user from his/her primary task, which could impair user's performance or comfort [24], [25]. In this respect, designing for short interactions (and interruptions) seems key for flexible timesharing between concurrent activities [26]. These results suggest that micro-interactions, multi-tasking, supporting actions in the real world, and the context of use will all be important elements influencing the use of HWCs. However, the specificity of the form-factors involved also raise the question of which interaction techniques are appropriate.

\section{B. Methodology}

Olsson et al. [27] reviewed a large range of methodologies for studying the user-experience of mobile augmented-reality applications. The authors conclude that an in-depth and qualitative study is necessary to understand how to remedy specific usability issues. In order to understand the current usage of HWCs we can adopt techniques from studies of mobile devices. From a methodological perspective, Müller et al. [18] used a combination of a diary study, interviews, and contextual inquiry observations for studying tablet use. Church et al. [28], who explored why and how people are using mobile search in social settings used a combination of a survey, a two-week diary study and follow-up interviews. Similarly, to identify the requirements for future HWCs, we adopted these methods for studying these devices in more detail. In the next section, we describe our methodology and results.

\section{UNDERSTANDING HEAD-WORN COMPUTERS}

We surveyed current users of HWCs regarding their usage patterns, intentions, and interactions. We followed a three-step approach consisting of (1) an online survey, (2) interviews with expert users, and (3) a diary study with novices. This was in order to investigate both novice and expert users and to explore their usage patterns and activities, environment, and interaction with the device. While the survey allowed us to get an overview and to understand the big picture, the other two methods gave us insights into specific use cases. 


\section{A. Method}

We used a triangulated research approach. Based on the AEIOU-framework ${ }^{9}$, our research covered the usage of the device, activities and applications, properties of the device itself, the environment in which the device is used, interaction techniques, and the user. In the following, we will focus on the activities, the environments and the interaction techniques.

For the online survey, we used a mixture of closed questions with five point Likert scales (e.g. $1=$ Totally unacceptable $-5=$ Totally acceptable, $1=$ Strongly disagree $-5=$ Strongly agree), and open questions for further feedback.

The expert interviews were semi-structured and lasted approximately one hour [29]. First, we introduced the design problem and the interview procedure. To get deeper insights we asked participants to report about their day before (overview question). We asked them to report on the five most common activities (demonstration of activities), cf. survey results. We asked for examples and clarification (looking for gaps), and investigated relationships, frustrations, background and goals of the participants (details as needed). Finally, we asked them, what they could imagine for the future in terms of functionality and interaction techniques.

Diary participants got a one-hour introduction and a handout with a more detailed explanation by following the approach of Carter and Mankoff [30]: For the in situ capture, participants took a picture or a note and shared them via a predefined Twitter account. In situ annotations were made by adding a caption to the picture and more extensive annotation was possible via smartphone or/and a desktop computer. Participants got push notifications with daily tips about what for, how and where they could use the device, and were encouraged to make entries regarding User Needs, Successes \& Failures as well as Barriers \& Problems. After three weeks, we conducted interviews to discuss their experience.

\section{B. Participants}

In total, we had 59 participants from across the world (North America, Europe, and Asia).

The survey was published in a number of social media forums related to smart glasses. We attracted 48 participants (one female) from North America (84\%), Europe (10\%) and Asia $(6 \%)$. Their ages ranged from 18 to 72 years $(M=39, S D$ = 12). 47 participants used Google Glass and one used an Oakley Airwave. 52\% of the participants reported to have less than one year of experience, $42 \%$ had $1-3$ years of experience, and $6 \%$ had more than three years of experience. $50 \%$ of the participants stated that they used their HWC 5+ times a day. $42 \%$ of the participants used their HWC 5+ hours a day, $21 \%$ 3-5 hours a day, 21\% 1-3 hours a day and 16\% less than one hour a day. The experience with the device ranged from 1 to 21 months ( $M=7$ months).

For the interviews, we recruited six experienced expert users (all male and all from the US) of HWCs. Their age ranged from 22 to 63 years $(M=35, S D=15)$ and they all had more than six months experience with the device. Two had a tech-

\footnotetext{
${ }^{9}$ http://help.ethnohub.com/guide/aeiou-framework
}

nical background, two a background in education, one was working in marketing, and one in government. All of them wore a Google Glass at the time of the interview. Two had also tried a Recon Jet before.

Our diary participants were four novel Google Glass users (one female) from our hometown. Their age ranged from 25 to 40 years $(M=32, S D=6)$. Three had a technical background, and made frequent use of their smartphone, tablet, desktop or laptop computer.

In summary, we tried to attract a broad range of users with different demographics. Nevertheless, the field of head-worn computers which are designed for everyday use is a very new and emerging field. Device access is partly limited to selected interested persons; therefore, survey participants were mostly male and technology affine persons. We suspect users involved in the study reflect the group of early adopters. Another fact is that most of the participants used a Google Glass during the period of the study, while only a few were experienced with other HWCs. However, we think that many of the results are applicable for other HWCs, which were designed for everyday use. For certain, aspects such as the social acceptability of HWCs are also dependent on the form factor of the device and the interaction techniques.

\section{Data Analysis}

To analyze the huge amount of data, we did quantitative analysis and used the grounded theory approach based on [29] for the qualitative data from the interviews and the diary studies.

For the survey, we did quantitative data analysis by calculating acceptance rates and performing Mann-Whitney-U tests for significance tests ${ }^{10}$. In order to evaluate the interview data, we started with a single-case analysis and broke the data down into AEIOU categories. Next, we did a cross-case analysis and searched for patterns and relationships across the participants. Additionally, we aggregated all the notes in an affinity diagram grouping it with the following properties: typical usage, activities, navigation, interaction techniques, social acceptability and reasons for/against HWCs. For the diary study, we used quantitative methods for the completed post-test questionnaires (the same as in the survey) and decoded the 112 diary entries by three different coding schemes: (1) type of diary entry (Problems and Barriers, Successes, Failures, User Needs), (2) AEIOU category, and (3) description of each item (Communication, Setup, Interaction Techniques, etc.). We followed the same approach for the interview notes and for the notes of the debriefing interviews after the diary studies.

\section{RESULTS \& DISCUSSION}

\section{A. Activities}

Based on common activities performed on tablets [18], we created a list of 22 activities, which can be performed on HWCs. We included activities which can also be performed on HWCs and excluded those which cannot be done on these

\footnotetext{
${ }^{10}$ We tested two independent groups (those who used the device (a) less than three hours a day - short-span users and (b) three or more hours a day long-span users) regarding their acceptance concerning e.g. environments.
} 
devices. We asked participants for frequency of use. Fig. 2 shows the top ten most frequently performed activities. Most of these activities were either related to short actions (taking pictures, recording videos), consuming content (viewing pictures, reading messages), getting information (getting directions, searching for information) or checking habits (checking the time / weather / calendar), cf. [21]. Activities like playing games or watching videos were less frequently performed, which was also confirmed by the diary study and the expert interviews.

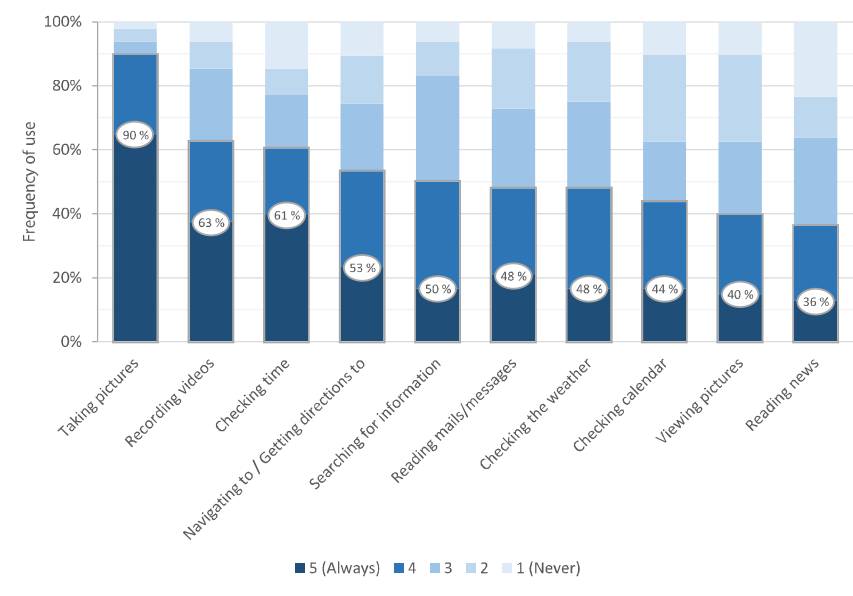

Fig. 2: Frequency of use of the top-ten activities.

The analysis of the top activities showed also that seven of the top-ten activities were context-sensitive - either influenced by location, identity, activity and/or time [31], e.g., getting directions by location, checking calendar by identity, searching for information by activity, checking the weather by time. For example, our interviewees liked to get tiny pieces of information when they were in motion. News, calendar entries or location-dependent information kept them up-to-date and enhanced their memory and their environment. They discovered buildings or places they had not seen before. More importantly, they just got information which was important to them at that instant in time.

\section{"It's all about information awareness. That you get the right package of information at the right time. Just small things instead of big issues." I5}

We also analyzed the according interaction steps to perform each of the top ten activities and found that all of them required one to three interaction steps to be performed in contrast to activities like playing games or social networking, which were less frequently performed and require many more interaction steps. This indicates that activities with a small number of interaction steps are more desirable. This was confirmed by the interviewees as they mentioned that they used the device for activities which are done quickly with low mental demand and physical effort. For example, participants mentioned that they were able to read messages in contexts where it was not possible before (e.g. while biking), because of the low cognitive effort. Another interesting finding was that novices focus more on very short actions or checking actions and experts make more use of trickier interactions like getting directions to or searching for information were they can request information on demand.
Additionally, we found during the expert interviews that the activities themselves have changed. One general observation was that users think much longer about the message, before they start to record it. This is because the effort to correct a message on a device like Google Glass is quite high as the only way to enter text is through speech input.

\section{"My messaging behavior has changed in the manner that I am quicker and my messages are shorter." I6}

However, all participants stated that they write shorter messages and they feel that the device make them more efficient.

"The length of the text message is as long it needs to be." I2

Messages were typically one to two sentences long. But participants also started creating workarounds to extend messages, like sending a second message. One common pattern was to use the HWC only for reading and a smartphone for writing.

Another interesting finding was that the device provides good support for documentation and instructional purposes. Participants recorded complete instructions (e.g. for cooking or repairing a bike) or captured feedback to give to others with minimal effort.

"I recorded that something went wrong during parking my car and sent it to my car manufacturer." I3

\section{B. Environments}

Our research objective for examining the environments was to understand the acceptance to use HWCs besides different audiences and in different spaces.

Our findings concerning the audiences were in line with work about gesture-based mobile interfaces from Rico and Brewster [32]. However, the overall acceptance rate for HWCs was noticeably higher in our study than in the earlier study. This can either be due to the fact that the acceptance for mobile devices in general has increased over the last years, or that HWCs are more acceptable than earlier gesture-based mobile interfaces. Participants were also more comfortable using the device around people that they knew.

The investigation of the spaces showed a general high acceptance rate $(M=74 \%)$ for device usage. Nevertheless, noisy environments had with $46 \%$ a lower acceptance rate as shown in Fig. 3. Participants mentioned that speech recognition was limited in environments like concerts/bars or while driving a car with open windows.

By comparing users ${ }^{11}$ who used the device less than three hours a day (short-span users) with those, who use it three or more hours a day (long-span users), we found that the first group preferred protected and private environments. For public, dynamic, noisy and both indoor and outdoor environments analysis revealed a significantly lower acceptance for shortspan users than for long-span users (see Fig. 3). For sure, those who are using the device for longer periods are more likely to use the device outdoor, in public or dynamic envi-

\footnotetext{
${ }^{11}$ Mann-Whitney-U test, $\mathrm{p}<.001$.
} 
ronments. The longer they used the device, the more familiar they became with the device and the more comfortable they are with specific environments. Moreover interviewees as well as diary participants reported using the device more in their leisure time (e.g. when they were on-the go or during sport activities) than at work, which reflects that they use it more in their private space, which can be also derived from the current frequent performed activities.

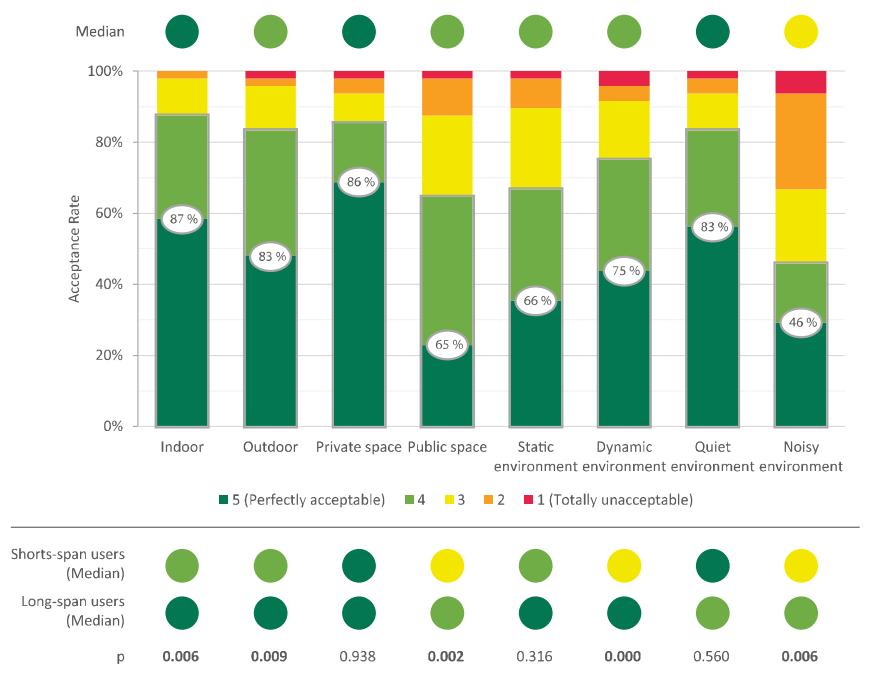

Fig. 3: Acceptance of device usage in different environments.

\section{Interaction}

In order to understand current interaction techniques, we investigated frequency, ease of use, and the desire for using them. Fig. 4 shows the frequency rate, ease of use rate, desire of use rate ${ }^{12}$ as well as the Medians for each interaction technique.

The social acceptability of the different interaction techniques was explored by asking the participants about their compliance with the four reasons [32] why gestures are most probably noticed as being uncomfortable: they look "weird", they are physically uncomfortable, there is interference with communication, and they are an uncommon movement.

\section{1) Eye gestures}

We found that eye gestures were strongly accepted by participants of all three studies. As Fig. 4 shows, eye gestures were frequently used, were perceived as easy to use and were desired to be used. The social acceptability was also high due to the fact that they are less obtrusive. On one side, participants liked that fact, but on the other side, bystanders struggle with recognizing these kind of gestures - especially if sun glasses were worn together with the HWC. Therefore, much more investigation would be necessary to explore the social acceptability according to bystanders. However, diary study participants mentioned that extending eye gestures could provide more hands-free interaction, if there is a possibility to overcome their limitations.

\footnotetext{
${ }^{12}$ Frequency rate $(5=$ Always, $4=$ Often $)$, Ease of use rate $(5=$ Very easy, $4=$ Easy) and Desire of use rate $(5=$ Very desirable, $4=$ Desirable). All positive answers aggregated.
}

\section{2) Head gestures}

In contrast to eye gestures, head gestures are more obtrusive. The social acceptability was neutrally rated. Head gestures were typically used for simple actions like checking the time. For complex interactions like playing a game with head gestures, participants reported strong concerns regarding the comfort to perform these gestures.

\section{3) Speech}

Both the ease and the desire of using speech got high ratings with Medians of 5. Interestingly, speech was more desired than touch gestures, which were more frequently performed and also easier to perform. In the diary study we found a possible explanation for this as all participants found speech input at least partly challenging. Entering addresses, writing messages or navigating hands-free through the menu was hard as the wording was often wrongly spelled. The level of noise in environments like restaurants also influenced the voice recognition of the device.

\section{"just had lunch difficult to operate the system with speech recognition" D1, Day 2}

\section{4) Touch gestures}

Touch gestures were most frequently used by the participants. The ease and desire of use as well as the social acceptability were quite high. Nevertheless, interviewees and diary participants mentioned some concerns regarding touch gestures. Interviewees mentioned that using the touchpad in motion (e.g. running) is quite hard as it requires a more precise interaction. In the diary study, we also found that participants struggled with the navigation concept, the mapping of the swiping directions and had especially troubles in the beginning. After the three weeks, all participants were able to interact with touch gestures. What we also found is that the anatomy of a person influences the ease of using this interaction technique. For example, one participant had long loose hair and had problems to reach and to interact with the touchpad.

\section{5) Interaction with an external device}

Currently, some devices let users control their HWC by using an additional control unit or a smartphone (e.g. Epson Moverio). These devices were rarely used for the interaction with a HWC (see Fig. 4). While the ease of use is a little lower, the desire of use and the social acceptability are quite high. To make quantifiable statements a much more detailed investigation of concrete external devices would be necessary. Currently, only a limited group of HWCs allows a control via an external device.

\section{6) Mid-air hand gestures}

The low preference for mid-air hand gestures (see Fig. 4) was confirmed and clarified by the interview results. Participants liked to use the device hands-free, which means also without any mid-air hand gestures. For example, they enabled the device by lifting the head, used eye gestures for taking pictures and speech for everything else - all hands-free.

"I think benefits of head-worn computers are that you can interact hands-free faster and easier." II 
Also those who used the device mostly with touch gestures mentioned situations where they interacted hands-free, such as in winter, when they usually have their hands in their pockets. Regarding the social acceptability, their main concerns were that the interaction looks weird and is an uncommon movement. In the interviews, we found that participants also use the device often on-the go. Therefore they need an unobtrusive way of interaction, which can be done in motion and does not interfere with any bystanders. In consideration of these requirements, as long as users need their hands for something else, mid-air hand gestures are hard-to-perform. Despite the context of use, the ease and the desire of using mid-air hand gestures are important for developing future mid-air hand gestures for HWCs.
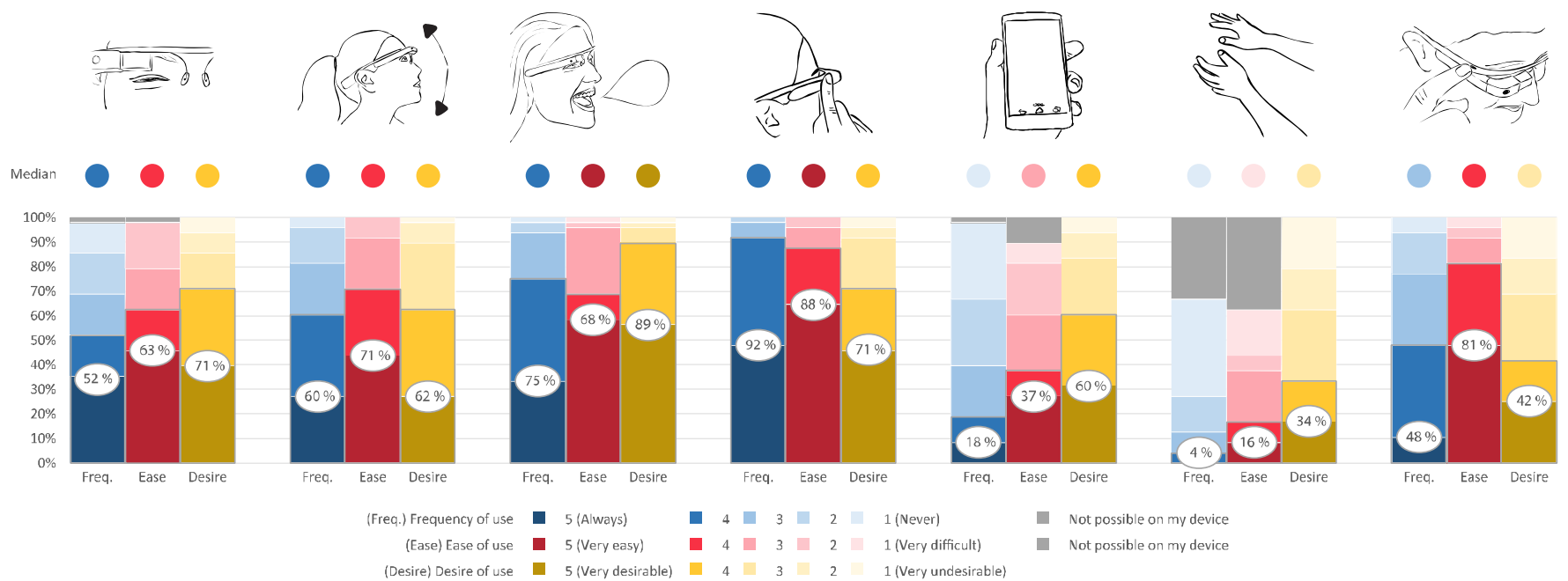

Fig. 4: Frequency of use, Ease of use, Desire of use for the different interaction techniques.

\section{7) Interaction with hardware buttons}

While hardware buttons were easy to use and had a high social acceptability, the level of desire of use and current frequency of use is only moderate. Participants reported that pushing a hardware button mounted on the device moves also the device itself, which can be uncomfortable.

\section{CONCLUSION AND FUTURE WORK}

In this paper, we provided insights into the use of HWCs by capturing the opinion of novice and expert users with a survey, interviews, and a diary study. Our research provides a detailed breakdown of activities performed on HWCs, explores the social acceptability in a variety of spaces and for users amongst different audiences, and investigates the various interaction techniques. This work is the first of its type to survey usage patterns of HWCs in the context of everyday use.

Our results showed that HWCs are primarily used for micro interactions and the provision of small pieces of information. Furthermore, we have seen that the most frequently performed activities only require little cognitive and physical load (e.g. checking the time, recording videos, searching on a map, or searching for information etc.). Our results are in line with Ganapathy et al. [33], who pointed to users preferring a minimal number of interaction steps, as long as necessary information could be displayed in one step. HWCs with controls situated in front of the user's eye provide the potential for easy and immediate access and overall shorter interaction times than mobile devices such as smartphones. This would offer an advantage for users while performing secondary activities, as the time it takes to resume their primary task would be lessened. Therefore, a HWC should support immediate access to the device such that users' can perform, for example, interactions on-the go and quickly ${ }^{13}$. In conclusion, we can confirm that designers should design for micro interactions ${ }^{14}$ [23] as we have seen that activities considering this are the most popular ones at the moment.

Concerning the environment, we can summarize that private and protected environments are preferred and users like to use the device around people that they know. In accordance with our results, many activities frequently performed on HWCs were context-sensitive (e.g. checking a grocery list while shopping, using a navigation system while driving, checking the weather before going outdoors). This is in line with Sohn et al. [22], who reported that $72 \%$ of the tasks on mobile devices were triggered by their context. Obviously, this can influence the interface, the content, or the interaction for a specific activity. A navigation and mapping application, for instance, should change its layout depending on the environment, e.g. if it is used in the subway, in a car, or walking down the street. All three environments have different properties. So, both the level of detail and the content have to be adjusted accordingly. Alongside the user interface, the interaction has to be considered and optimized according to the context, in which the user is at the moment (e.g. touch gestures while running can become difficult and could be improved by speech input or other ways of interaction). Hence, we can conclude that we should design for the device and the current context ${ }^{10,15}$.

\footnotetext{
${ }^{13}$ https://developer.android.com/design/wear/principles

${ }^{14}$ http://de.slideshare.net/marknb00/siggraph-2014-the-glass-classdesigning-wearable-interfaces

${ }^{15}$ https://developers.google.com/glass/design/principles
} 
The investigation of the interaction techniques showed that touch gestures were most frequently used, while speech is most desired. Indeed, we found that one of the key benefits of the device is hands-free interaction. For example, participants liked to use the device for secondary actions, while they were driving, repairing things, taking care of kids, or cooking. Eye gestures, head gestures or speech point already in that direction. However, there is still potential to explore the field of hands-free interaction techniques in order to support inattentive interaction, which can highlight the benefits of HWCs.

This paper just touches the surface of a very exciting new field of wearables and shed more light on key aspects of the everyday use of HWCs. In turn, those aspects call for exploring further some particular issues such as innovative input techniques which support micro-interactions and contextsensitivity. This includes developing new hands-free, imprecise or inattentive interactions techniques [34] that will reduce the complexity of device interaction. Our results showed that users may be reluctant to use applications requiring a high number of interaction steps or even adapt their behavior accordingly (cf., messaging). This will be essential for enabling users to get the most out of the device in activities like messaging or accessing documentation and instructions. Finally, our study could be further validated with a longer longitudinal study encompassing different cultural groups.

\section{ACKNOWLEDGMENTS}

The eGlasses consortium receives the funding support of NCBiR, FWF, SNSF, ANR, and FNR in the framework of the ERA-NET CHIST-ERA II.

\section{REFERENCES}

[1] I. E. Sutherland, "A head-mounted three dimensional display," in AFIPS, 1968, p. 757.

2] L. Blum, R. Wetzel, R. McCall, and L. Oppermann, "The fina TimeWarp: Using Form and Content to Support Player Experience and Presence when Designing Location-AwareMobile Augmented Reality Games," in DIS, 2012, pp. 771-720.

[3] S. Feiner, B. MacIntyre, T. Höllerer, and A. Webster, "A touring machine: Prototyping 3D mobile augmented reality systems for exploring the urban environment," in Personal Technologies, 1997, vol. 97, pp. 74-81.

[4] B. Thomas, V. Demczuk, W. Piekarski, D. Hepworth, and B. Gunther, "A wearable computer system with augmented reality to support terrestrial navigation," in $I S W C, 1998$, pp. 168-171.

[5] B. Rhodes, "The wearable remembrance agent: A system for augmented memory," Pers. Technol., vol. 1, no. 4, pp. 218-224, 1997.

[6] D. Drascic and P. Milgram, "Perceptual Issues in Augmented Reality Reality-Virtuality Continuum," Virtual Real., vol. 2653, pp. 123-134, 1996.

[7] R. Azuma, R. Behringer, S. Feiner, S. Julier, and B. Macintyre, "Recen Advances in Augmented Reality," Comput. Graph., vol. 21, no. November, pp. 1-15, 2001.

[8] M. A. Livingston, J. L. Gabbard, J. E. Swan II, C. M. Sibley, and J. H. Barrow, "Basic Perception in Head-Worn Augmented Reality Displays," in Human Factors in Augmented Reality Environments, W. Huang, L. Alem, and M. A. Livingston, Eds. New York: Springer New York, 2013 pp. 109-135

[9] U. Neumann and A. Majoros, "Cognitive, performance, and systems issues for augmented reality applications in manufacturing and maintenance,"VR, 1998.

[10] L. Nigay, P. Salembier, T. Marchand, P. Renevier, and L. Pasqualetti, "Mobile and Collaborative Augmented Reality: A Scenario Based
Design Approach," in Human Computer Interaction with Mobile Devices, F. Paternò, Ed. Berlin, Heidelberg: Springer Berlin Heidelberg, 2002, pp. 241-255.

[11] J.-Y. Didier, D. Roussel, M. Mallem, S. Otmane, S. Naudet, Q.-C Pham, S. Bourgeois, C. Mégard, C. Leroux, and A. Hocquard, "AMRA : Augmented Reality assistance in train maintenance tasks," ISMAR, no. Ismar, pp. 1-10, 2005.

[12] A. Dünser, R. Grasset, and M. Billinghurst, "A survey of evaluation techniques used in augmented reality studies," Canterbury, 2008.

[13] J. E. Swan II and J. L. Gabbard, "Survey of User - Based Experimentation in Augmented Reality," VR, pp. 1-9, 2005.

[14] H. Beyer and K. Holtzblatt, Contextual design: defining customercentered systems. Morgan Kaufmann Pub, 1998.

[15] T. E. Starner, "Wearable Computing and Contextual Awareness," Massachusetts Institute of Technology, 1999.

[16] K. Lyons, "Everyday Wearable Computer Use: A Case Study of an Expert User," Human-Computer Interact. with Mob. Devices Serv., vol. 2795, pp. 61-75, 2003.

[17] I. Pedersen and D. Trueman, "'Sergey Brin is Batman,"” in CHI EA, 2013, p. 2089.

[18] H. Müller, J. Gove, and J. Webb, "Understanding tablet use," in MobileHCI, 2012, p. 1.

[19] M. McGregor, B. Brown, and D. McMillan, "100 days of iPhone use," in CHI EA, 2014, pp. 2335-2340.

[20] M. Böhmer, T. S. Saponas, and J. Teevan, "Smartphone use does not have to be rude," in MobileHCI, 2013, p. 342.

[21] A. Oulasvirta, T. Rattenbury, L. Ma, and E. Raita, "Habits make smartphone use more pervasive," Pers. Ubiquitous Comput., vol. 16, no. 1, pp. 105-114, Jun. 2011.

[22] T. Sohn, K. a. Li, W. G. Griswold, and J. D. Hollan, "A diary study of mobile information needs," in CHI, 2008, p. 433.

[23] D. L. Ashbrook, "Enabling mobile microinteractions," Georgia Institute of Technology, 2010

[24] T. Gillie and D. Broadbent, "What makes interruptions disruptive? A study of length, similarity, and complexity," Psychol. Res., vol. 50, no. 4, pp. 243-250, 1989

[25] D. C. McFarlane and K. A. Latorella, "The Scope and Importance of Human Interruption in Human-Computer Interaction Design," HumanComputer Interact., vol. 17, no. 1, pp. 1-61, 2002.

[26] D. D. Salvucci, N. a. Taatgen, and J. Borst, "Toward a unified theory of the multitasking continuum: From concurrent performance to task switching, interruption, and resumption," in CHI, 2009, pp. 1819-1828.

[27] T. Olsson, "Concepts and Subjective Measures for Evaluating User Experience of Mobile Augmented Reality Services," in Human Factor in Augmented Reality Environments, W. Huang, L. Alem, and M. A. Livingston, Eds. New York: Springer New York, 2013, pp. 203-232.

[28] K. Church, A. Cousin, and N. Oliver, "I wanted to settle a bet!," in MobileHCI, 2012, no. December 2011, p. 393.

[29] K. Goodwin, Design for the Digital Age: How to Create HumanCentered Products and Services. Wiley Publishing, 2009.

[30] B. A. T. Brown, A. J. Sellen, and K. P. O'Hara, "A diary study of information capture in working life," in $\mathrm{CHI}, 2000$, pp. 438-445.

[31] G. Abowd and A. D. Dey, "Towards a better understanding of context and context-awareness," Comput. Syst., vol. 40, no. 3, pp. 304-307, Nov. 1999.

[32] J. Rico and S. Brewster, "Usable gestures for mobile interfaces," in $C H I$, 2010 , p. 887.

[33] S. Ganapathy, G. J. Anderson, and I. V. Kozintsev, "Empirical evaluation of augmented information presentation on small form factors - Navigation assistant scenario," in ISVRI, 2011, pp. 75-80.

[34] S. E. Hudson, C. Harrison, B. L. Harrison, and A. LaMarca, "Whack gestures," in TEI, 2010, p. 109. 